\title{
Transcriptional regulation of anthocyanin biosynthesis in a high-anthocyanin resynthesized Brassica napus cultivar
}

\author{
Gayatri Goswami ${ }^{1 \dagger}$, Ujjal Kumar Nath ${ }^{1,2 \dagger}$, Jong-In Park ${ }^{1 *}$, Mohammad Rashed Hossain 1,2, \\ Manosh Kumar Biswas ${ }^{1}$, Hoy-Taek Kim ${ }^{1,3}$, Hye Ran Kim ${ }^{4}$ and III-Sup Nou ${ }^{1 *}$
}

\begin{abstract}
Background: Anthocyanins are plant secondary metabolites with key roles in attracting insect pollinators and protecting against biotic and abiotic stresses. They have potential health-promoting effects as part of the human diet. Anthocyanin biosynthesis has been elucidated in many species, enabling the development of anthocyanin-enriched fruits, vegetables, and grains; however, few studies have investigated Brassica napus anthocyanin biosynthesis.

Results: We developed a high-anthocyanin resynthesized B. napus line, Rs035, by crossing anthocyanin-rich B. rapa (A genome) and B. oleracea (C genome) lines, followed by chromosome doubling. We identified and characterized 73 and 58 anthocyanin biosynthesis genes in silico in the $A$ and $C$ genomes, respectively; these genes showed syntenic relationships with 41 genes in Arabidopsis thaliana and B. napus. Among the syntenic genes, twelve biosynthetic and six regulatory genes showed transgressively higher expression in Rs035, and eight structural genes and one regulatory gene showed additive expression. We identified three early-, four late-biosynthesis pathways, three transcriptional regulator genes, and one transporter as putative candidates enhancing anthocyanin accumulation in Rs035. Principal component analysis and Pearson's correlation coefficients corroborated the contribution of these genes to anthocyanin accumulation.
\end{abstract}

Conclusions: Our study lays the foundation for producing high-anthocyanin B. napus cultivars. The resynthesized lines and the differentially expressed genes we have identified could be used to transfer the anthocyanin traits to other commercial rapeseed lines using molecular and conventional breeding.

Keywords: Brassica napus, Resynthesized, Microsynteny, Transgressive expression, Additive expression, Anthocyanins

\section{Background}

Anthocyanins belong to the flavonoid class of secondary metabolites. These water-soluble pigments are widely distributed in plants, accumulating in the leaves, petals, sepals, and fruits to yield purple, red, and blue coloration $[1,2]$. Anthocyanins attract insects for pollination and protect plants against biotic and abiotic stresses [3-7]. Eating foods enriched with anthocyanins might reduce inflammation and protect against certain types of cancer,

\footnotetext{
*Correspondence: jipark@sunchon.ac.kr; nis@sunchon.ac.kr

${ }^{\dagger}$ Gayatri Goswami and Ujjal Kumar Nath contributed equally to this work

${ }^{1}$ Department of Horticulture, Sunchon National University, 255

Jungang-ro, Suncheon, Jeonnam 57922, South Korea

Full list of author information is available at the end of the article
}

cardiovascular, neurodegenerative, and various agerelated diseases [8-10]. The health benefits of anthocyanins are believed to be closely linked to their antioxidant activities, through which they reduce the abundance of reactive oxygen species [11]. Anthocyanins also increase blood serum antioxidant levels and protect red blood cells from oxidative damage $[8,12]$.

Brassica napus is a natural allopolyploid with the genomic composition $2 \mathrm{n}=4 \mathrm{x}=38$ (AACC). In addition to its international use as an oilseed and fodder crop, it is becoming increasingly popular as a vegetable, and a number of $B$. napus vegetable varieties have been developed [13]. For example, the leaves and edible flower buds and young stems of $B$. napus are consumed as vegetables 
in China and other Asian countries, and one subspecies is also commonly used as a root vegetable in Europe, the U.S., Canada, and Australia (rutabaga, B. napus subsp. rapifera). Considering the diverse health-promoting benefits of anthocyanins, $B$. napus cultivars with high anthocyanin contents may be popular with consumers; however, only one study has documented $B$. napus mutants with red or purple leaves [14]. In contrast, several high-anthocyanin varieties are available for two of the most popular leafy Brassicaceae vegetables, Chinese cabbage (B. rapa ssp. pekinensis) and cabbage (B. oleracea var. capitata).

Understanding the regulation of anthocyanin biosynthesis is vital for enabling us to develop anthocyaninenriched fruits and vegetables. Anthocyanin biosynthesis begins in the cytoplasm under the coordinated regulation of the structural genes, which encode several enzymes and regulatory transcription factors [15]. Glutathione $S$-transferases are involved in the export of the anthocyanins from the cytoplasm to the vacuole, where they are permanently stored $[16,17]$. The anthocyanin biosynthesis pathway can be divided into three major consecutive phases (Fig. 1). In the first phase, phenylalanine is converted into trans-cinnamic acid and 4-coumaroyl-CoA by phenylalanine ammonia-lyase (PAL), cinnamate 4-hydroxylase $(\mathrm{C} 4 \mathrm{H})$, and 4-coumarate-CoA ligase (4CL), respectively [18-20]. Next, a series of enzymatic reactions catalyzed by chalcone synthase (CHS), chalcone isomerase (CHI), flavanone-3-hydroxylase $(\mathrm{F} 3 \mathrm{H})$ lead to the formation of dihydroflavonol from one molecule of coumarate-CoA and three molecules of malonyl-CoA. In the third stage, many types of anthocyanidins are formed from the dihydroflavonols, in reactions catalyzed by dihydroflavonol 4-reductase (DFR) and anthocyanidin synthase (ANS). Anthocyanidins are finally modified by a series of glycosylation and methylation steps to form anthocyanins, catalyzed by UDPglucose:flavonoid glucosyltransferase (UFGT) and methyl transferase (MT) [21, 22].

Both structural and regulatory genes are involved in anthocyanin accumulation in Arabidopsis thaliana [19, 23, 24]. Primarily, anthocyanin biosynthesis is regulated

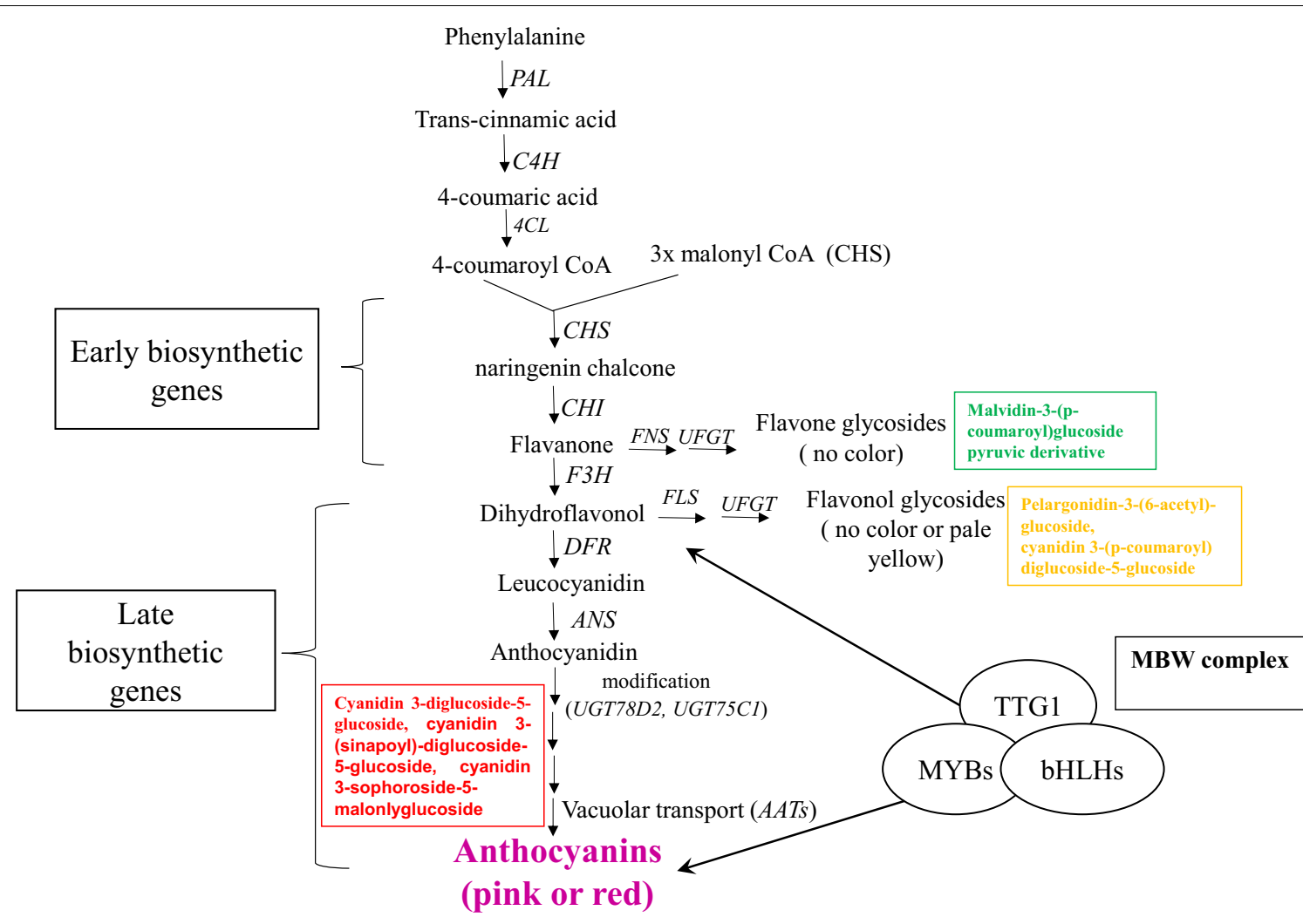

Fig. 1 Schematic and simplified diagram of the anthocyanin biosynthesis pathway leading to the accumulation of anthocyanin in Brassica. The pathway is divided into three major parts; the upstream phenylpropanoid pathway, the early biosynthetic pathway, and the late biosynthetic pathway, based on the types of genes involved in each stage. The possible activation sites of the regulatory MBW complexes are indicated. Details of the genes involved in each stage are provided in Table 2. PAL phenylalanine ammonia lyase, C4H cinnamate-4-hydroxylase, 4CL 4-Coumaratel-CoA ligase, CHS chalcone synthase, CHI chalcone isomerase, F3H flavanone-3-hydroxylase, FLS flavonol synthase, DFR dihydroflavanol reductase, ANS anthocyanidin synthase, UGT uradin glycosyltransferase, MYB myeloblastosis family of transcription factors, bHLH basic helix-loop-helix 
at the transcriptional level; previous studies demonstrated that the expression patterns of the anthocyanin structural genes are mainly determined by the R2R3MYB (myeloblastosis family of transcription factors), basic helix-loop-helix (bHLH), and WD40-repeat transcription factors, and by their coordinated interactions [25]. Lang et al. [26] reported that R2R3-MYB and bHLH transcription factors can bind to specific cis-acting elements in promoter regions of the structural genes and regulate their expression. The MBW (R2R3-MYB, bHLH, and WD40) complex is unique to plants, but this transcriptional regulatory system may vary between monocot and dicot plants; all three members of the MBW complex are not required for anthocyanin biosynthesis in every species $[21,22]$.

Recent studies have revealed that, in addition to the regulatory genes, the structural genes could also play a vital role in the accumulation of high levels of anthocyanin in several plant species [18, 27]. Zhang et al. [27] reported that most of the structural genes, including $P A L$, CHS, F3H, F3'H, DFR, ANS, and anthocyanin 5-0-glucosyltransferase (5GT) were significantly upregulated in purple kohlrabi (Brassica oleracea var. gongylodes), while only two regulatory genes, transparent testa8 (TT8) and production of anthocyanin pigment2 (PAP2) were upregulated. Previous reports showed that most of the anthocyanin pathway structural genes are upregulated in B. rapa varieties with high anthocyanin levels $[28,29]$. The increased expression of structural genes in red cabbage was found to be coordinated by a bHLH, TT8, and a MYB transcription factor gene, MYB2 [11].

Allopolyploidization, the merging of two or more divergent genomes into a common background, enhances the genomic diversity of the resulting organism [30-32]. Allopolyploids arise from interspecific or intergenic hybridization followed by chromosome doubling, and can act as a bridge species for gene introgression into important crop species. The genus Brassica contains a number of diploid and allopolyploid species. Among them, six agriculturally important species can be categorized into three basic diploid genomes (A, B, and $C ; n=10,8$, and 9, respectively) and their allopolyploid hybrids ( $\mathrm{AB}, \mathrm{AC}$, and $\mathrm{BC}$ ), as confirmed using cytogenetics [33]. Brassica napus (AACC; $2 \mathrm{n}=38$ ) is an allopolyploid that evolved following the hybridization of its ancestors B. rapa (AA; $2 \mathrm{n}=20)$ and B. oleracea $(\mathrm{CC}$, $2 \mathrm{n}=18)$ [33]. Sequence variations within chromosome segments showed that some of the A- and C-genome components of $B$. napus have undergone genetic changes since this hybridization event [34-36]. In contrast, resynthesized B. napus (produced by crossing B. rapa and $B$. oleracea followed by embryo rescue and chromosome doubling) was shown to undergo extensive chromosomal rearrangements immediately after allopolyploidization [37-39]. In the present study, we therefore found a wide variation in anthocyanin contents in resynthesized $B$. napus lines rather than either of the parents or intermediate of the parental lines.

Here, we characterized the structural and regulatory genes of anthocyanin biosynthesis in a purple-colored resynthesized $B$. napus line. We developed an anthocyanin-enriched allopolyploid $B$. napus by crossing a red-green Chinese cabbage (B. rapa ssp. pekinensis, AA genome) and a red cabbage (B. oleracea var. capitata, DD genome), followed by embryo rescue and chromosome doubling using colchicine. The aims of this study were to (1) select a stable anthocyanin-enriched resynthesized $B$. napus line developed by interspecies hybridization, (2) investigate the impact of the total anthocyanin content on the purple pigmentation of the leaves, (3) predict the candidate genes responsible for the purple leaf trait in $B$. napus using expression profiling and determine the expression patterns of those genes, and (4) determine the relationship between the different gene expression profiles and total and constituent anthocyanin contents in $B$. napus.

\section{Results}

\section{Selection of an anthocyanin-enriched resynthesized $B$.} napus line

The anthocyanin-enriched B. napus line was selected based on visual observations and spectrophotometric of the 39 resynthesized (Rs) lines developed by crossing a red-green Chinese cabbage inbred line (Asia) and a red cabbage inbred line (Rubea) followed by embryo rescue and chromosome doubling. Allopolyploids resulting from the interspecies cross were confirmed using the COS1078 marker and by checking their DNA contents using flow cytometry (data not shown). The Rs lines had a wide variety of total anthocyanin contents $(<0.5$ to $>4.5 \mathrm{mg} \mathrm{g}^{-1}$ FW) (Fig. 2). Five Rs lines with total anthocyanin contents $>4.0 \mathrm{mg} \mathrm{g}^{-1} \mathrm{FW}$ were selected and evaluated in a five-replicate trial during the next season. Of these, Rs035 had the highest total anthocyanin content $\left(5.13 \pm 0.17 \mathrm{mg} \mathrm{g}^{-1} \mathrm{FW}\right)$ and was selected for further expression profiling using qRT-PCR.

\section{Evaluation of the anthocyanin profiles in Brassica lines}

The total anthocyanin contents of contrastingly pigmented Brassica lines (Asia, Rubea, the red Rs line Rs035, and the green Rs line Rs306) were measured. The total anthocyanin contents were higher in the three red lines (Asia, Rs035, and Rubea; Additional file 5: Figure S1). The Rs035 line had 2.6-fold higher anthocyanin content compared to its B. rapa parent (Asia). Moreover, the same line had comparatively lower anthocynin than its $B$. 


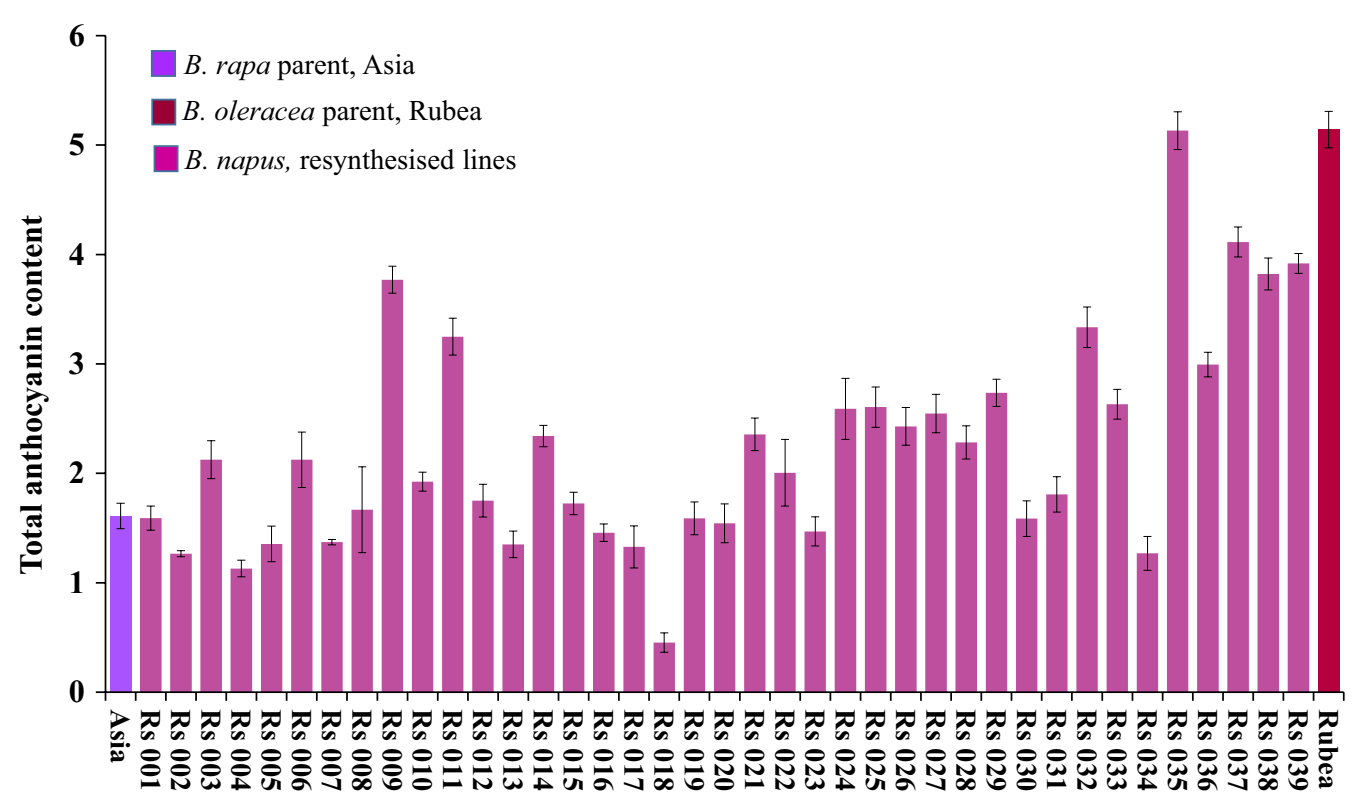

Genotypes

Fig. 2 Total anthocyanin contents in 39 resynthesized B. napus lines along with their parental lines

oleracea parent (cv. Rubea) (Fig. 2). No anthocyanin was detected in the resynthesized green B. napus line Rs306.

We carried out HPLC to investigate the anthocyanin composition of our contrasting Brassica plants. A total of six anthocyanin components were detected in the four lines (Table 1), of which cyanidin-3-diglucoside-5-glucoside, cyanidin-3-(sinapoyl)-diglucoside-5-glucoside, cyanidin-3-sophoroside-5-malonylglucoside, malvidin-3-( $p$-coumaroyl) glucoside pyruvic derivative, and pelargonidin-3-(6-acetyl)glucoside were the major anthocyanin components in Rs035. Four anthocyanin components were detected in the Chinese cabbage line Asia: cyanidin-3-diglucoside-5-glucoside, cyanidin-3-(sinapoyl)-diglucoside-5-glucoside, cyanidin- 3-sophoroside-5-malonylglucoside, and pelargonidin-3-(6acetyl)-glucoside. Among the six anthocyanin components, only three components, cyanidin-3-diglucoside-5-glucoside, cyanidin-3-(sinapoyl)-diglucoside-5-glucoside, and cyanidin-3-( $p$-coumaroyl)-diglucoside-5-glucoside, were found in the red cabbage line Rubea. None of the anthocyanin components were detected in the green Rs306 line (Table 1).

\section{Selection and in silico characterization of anthocyanin biosynthesis pathway genes}

To predict and characterize the putative anthocyanin biosynthesis candidate genes in resynthesized $B$. napus, the anthocyanin pathway genes in A. thaliana were used

Table 1 Anthocyanin composition in leaves of B. rapa reddish line Asia (A-genome), resynthesis green B. napus line Rs306, resynthesis red B. napus line Rs035 and B. oleracea red cabbage line Rubea (C-genome) as detected by HPLC

\begin{tabular}{|c|c|c|c|c|}
\hline \multirow[t]{2}{*}{ Anthocyanin component } & \multicolumn{4}{|c|}{ Anthocyanin levels ( $\mathrm{mg} \mathrm{g}^{-1}$ dry weight) } \\
\hline & Asia & Rs306 & Rs035 & Rubea \\
\hline Cyanidin3-diglucoside-5-glucoside & 0.041 & ND & 0.235 & 0.59 \\
\hline Cyanidin3-(sinapoyl)-diglucoside-5-glucoside & 0.105 & ND & 1.630 & 0.32 \\
\hline Cyanidin3-sophoroside-5-malonylglucoside & 0.032 & ND & 0.070 & ND \\
\hline Malvidin3-(p-coumaroyl)glucoside pyruvic derivative & ND & ND & 0.067 & ND \\
\hline Pelargonidin3-(6-acetyl)-glucoside & 0.262 & ND & 0.256 & ND \\
\hline Cyanidin3-(p-coumaroyl) diglucoside-5-glucoside & ND & ND & ND & 0.20 \\
\hline
\end{tabular}

$N D$ not detected 
to perform a syntenic gene search of the A (B. rapa) and $C$ (B. oleracea) genomes from the BRAD database. The Bolbase and EnsemblPlants databases were also searched for anthocyanin genes in these species. A complementary method, Hidden Markov Model profiling, was performed to increase the accuracy of the identified genes within the $B$. rapa and B. oleracea genomes. The chromosomal position and the sub-genome status of these putative anthocyanin biosynthesis pathway genes, as well as the isoelectric points $(\mathrm{Pi})$, molecular weights, and residual protein size of their predicted proteins, are presented in Additional file 1: Tables S1a and Additional file 2: Table S1b for B. rapa and B. oleracea, respectively.

We identified 73 putative anthocyanin pathway genes in B. rapa (A genome), of which 72 could be mapped onto one of its 10 chromosomes; chromosomes A01A10 contained 3, 12, 12, 7, 10, 4, 6, 1, 12, and 5 putative genes, respectively. The gene, Bra035004, an ortholog of UGT79B1, is located on Scaffold000100 and could not be assigned to a chromosome. Of the other 72 putative genes, 21 were structural biosynthetic genes in the phenylpropanoid pathway, 18 were involved in early biosynthesis, six were late biosynthesis genes, 12 were positive regulators, 13 were negative regulators, and two encoded transporters (Additional file 1: Table S1a and Additional file 5: Figure S2a).

In $B$. oleracea (C genome), a total of 58 anthocyanin biosynthesis pathway genes were identified, of which 37 could be assigned to one of the nine chromosomes; chromosomes C01-C09 contained 2, 6, 4, 4, 1, 3, 6, 5, and 6 of the putative genes, respectively (Additional file 1 : Table S1b and Additional file 5: Figure S2b). The other 21 genes were present on scaffolds, non-contiguous genomic sequences. The putative $B$. oleracea genes were categorized as 13 structural biosynthetic genes in the phenylpropanoid pathway, 13 early-biosynthesis genes, six late-biosynthesis genes, 24 positive and negative regulators, and two transporters (Additional file 2: Table S1b and Additional file 5: Figure S2b).

Three separate microsynteny maps were constructed using B. rapa, B. oleracea, and A. thaliana orthologous pairs of structural, regulatory, and transporter genes in the anthocyanin pathway to investigate their evolutionary cues and genetic relationships among the three species (Fig. 3a-c). Based on our analysis, 41 structural genes in B. rapa were found to correspond to 24 A. thaliana orthologous genes, while 32 orthologous gene pairs were recognized between $B$. rapa and B. oleracea (Fig. 3a). Of the regulatory genes, the 24 identified in $B$. rapa showed syntenic relationships with 16 orthologous genes in $A$. thaliana, while 24 pairs of syntenic regulatory genes were found between B. rapa and B. oleracea (Fig. 3b). Meanwhile, two syntenic transporter genes corresponding to an $A$. thaliana ortholog were identified in both $B$. rapa and $B$. oleracea (Fig. 3c). These results suggest that the structural, regulatory, and transporter genes of the anthocyanin pathway maintain close syntenic relationships in B. rapa, B. oleracea, and A. thaliana.

\section{Prediction of putative candidate genes for the enhanced accumulation of anthocyanin \\ Expression profiling of anthocyanin biosynthesis genes}

We carried out expression profiling of the anthocyanin biosynthesis genes to investigate their role in anthocyanin accumulation in the leaves of the contrasting Brassica lines. No striking effects were observed for the expression of the A-genome (B. rapa) and C-genome $(B$. oleracea) upstream (PAL1, PAL2, C4H, 4CL1) and downstream (UGT78D2) genes of the anthocyanin pathway in the four lines examined (Additional file 5: Figure S3a), despite their differences in anthocyanin accumulation. However, the downstream gene $U G T 78 D 2$ was upregulated in the A genome of Rs035, with a seven-fold higher expression than in the green line Rs306. This result is expected because of $U G T 78 D 2$ gene has a preference of Cyanidin3-(sinapoyl)-diglucoside-5-glucoside metabolism and found higher amount in Rs035 compared to Rubea (Table 1).

Among the upstream genes, $C H S, C H I, F 3^{\prime} H$, and $F L S 1$ showed 2-, 10-, 1.8-, and 20-fold higher levels of expression in the A genome of Rs035 than in its $\mathrm{C}$ genome, respectively, and were even 1.5-, 1.8-, 2-, and 10-fold more highly expressed than in the A-genome donor parental line, Asia (Fig. 4a). By contrast, $F 3 H$ gene showed 1.6-fold higher expression in the C-genome of Rs035 than its A-genome. These results indicate that the highly upregulated upstream genes in the $\mathrm{A}$ and $\mathrm{C}$ genomes of Rs 035 could be a major factor in its high accumulation of anthocyanin, although the genes from both the A and C genomes were upregulated compared to the donor parents, indicating a synergistic or positive effect of the two genomes overall. In contrast, the upstream gene $F 3 H$ in the $\mathrm{C}$ genome of Rs035 showed a 1.5-fold higher level of expression than that of in A-genome, and a 15-fold higher expression than in the $\mathrm{C}$-genome donor line, Rubea (Fig. 4a). The upstream gene $F 3^{\prime} H$ in the A genome of Rs035 had twofold higher expression compared to A-genome donor line, Asia. These results indicate that $F 3 H$ and $F 3^{\prime} H$ had a strong positive effect on enhancing the accumulation of anthocyanin accumulation in Rs035, but $\mathrm{A}$ and $\mathrm{C}$ genomes complemented each other.

The A-genome downstream anthocyanin biosynthesis genes DFR, ANS, and UGT75C1 were more highly expressed in Rs035 than their C-genome counterparts with 60-, 4-, and 55-fold higher expression levels, respectively. DFR, ANS, and UGT75C1 were expressed 5-, 3-, 

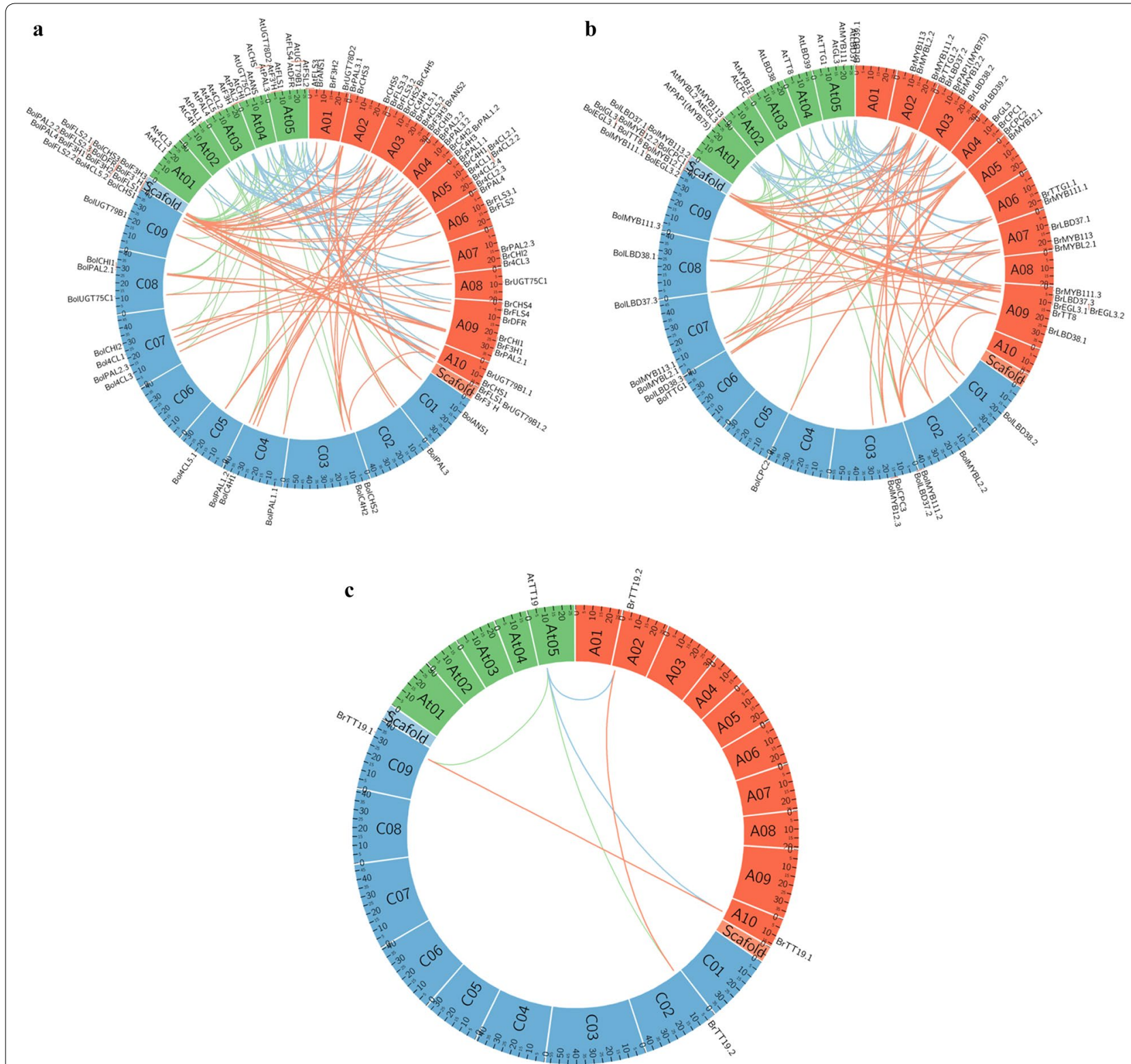

Fig. 3 Microsyntenic analyses of anthocyanin biosynthesis pathway genes. a Structural, early-biosynthesis, and late-biosynthesis genes, b positive- and negative-regulatory genes, and c transporter genes in Arabidopsis thaliana (At, green), Brassica rapa (A, orange), and B. oleracea (C, blue)

and 1.5-fold more highly in Rs035, respectively, than in the A-genome donor parent, Asia (Fig. 4b). Twelve biosynthetic genes (PAL1, PAL2, 4CL1, CHS, CHI, F3H, $F 3^{\prime} H$, UGT78D2, FLS1, DFR, ANS, and UGT75C1) showed a transgressive overexpression (significantly exceeding the expression levels of both parental species) in the resynthesized line Rs035 in A genome background (Fig. 4a, b and Additional file 5: Figure S3a). Moreover, eight structural and biosynthetic genes (PAL1, PAL2, $C 4 H, C H S, F 3^{\prime} H, F L S 1, D F R$, and $\left.A N S\right)$ showed higher expression in the resynthesized line Rs035 in C genome background compared to Rs035 in A genome background (Fig. 4a, b and Additional file 5: Figure S3a). The other structural genes of the $\mathrm{A}$ and $\mathrm{C}$ genomes showed non-additive expression. These results indicate that the highly expressed downstream genes (DFR, ANS, and UGT75C1) might be responsible for the enhanced accumulation of anthocyanin in Rs035, and that the expression of these genes is significantly upregulated by the interaction between the $\mathrm{A}$ and $\mathrm{C}$ genomes. 


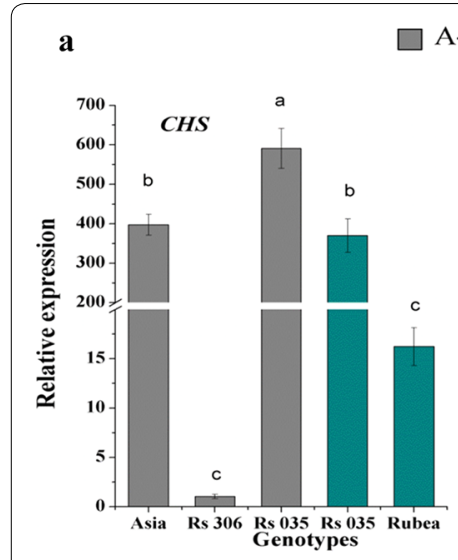

A-genome background

C-genome background
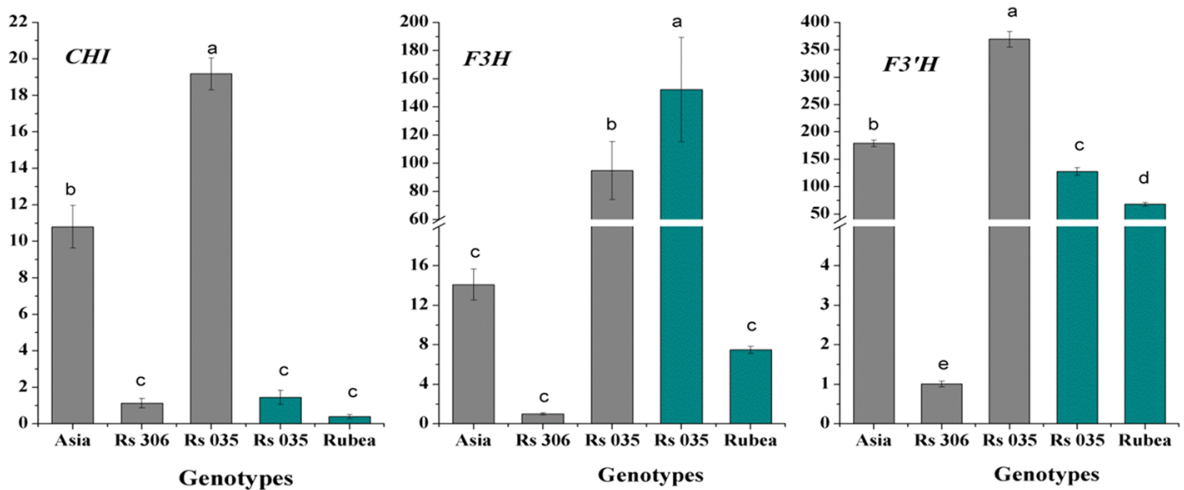

b
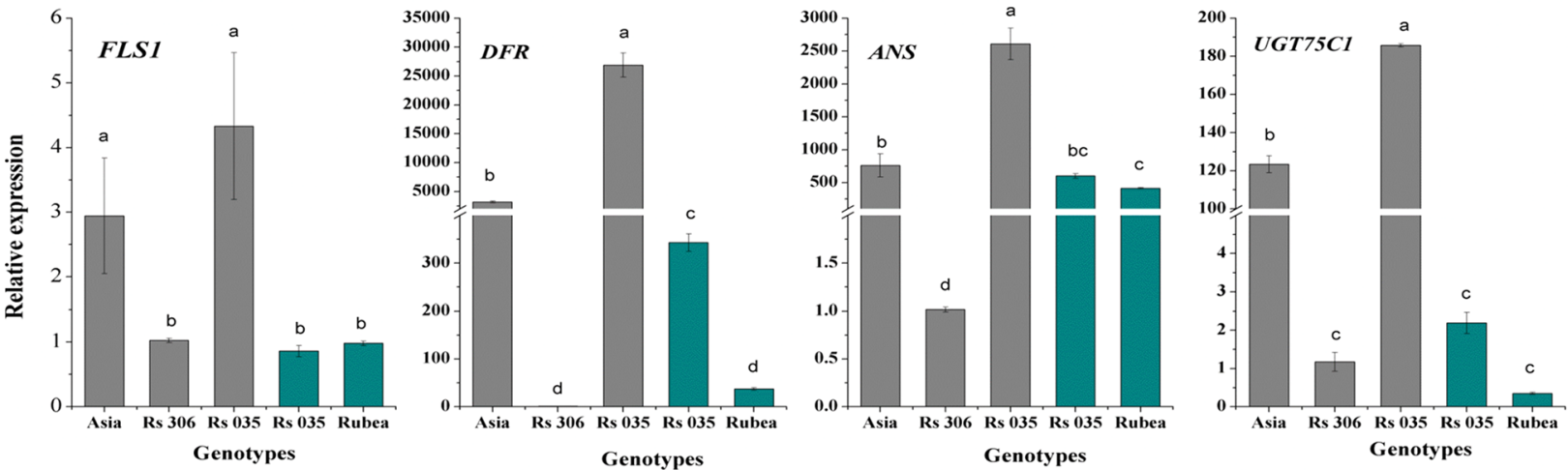

A-genome background $\square$ C-genome background
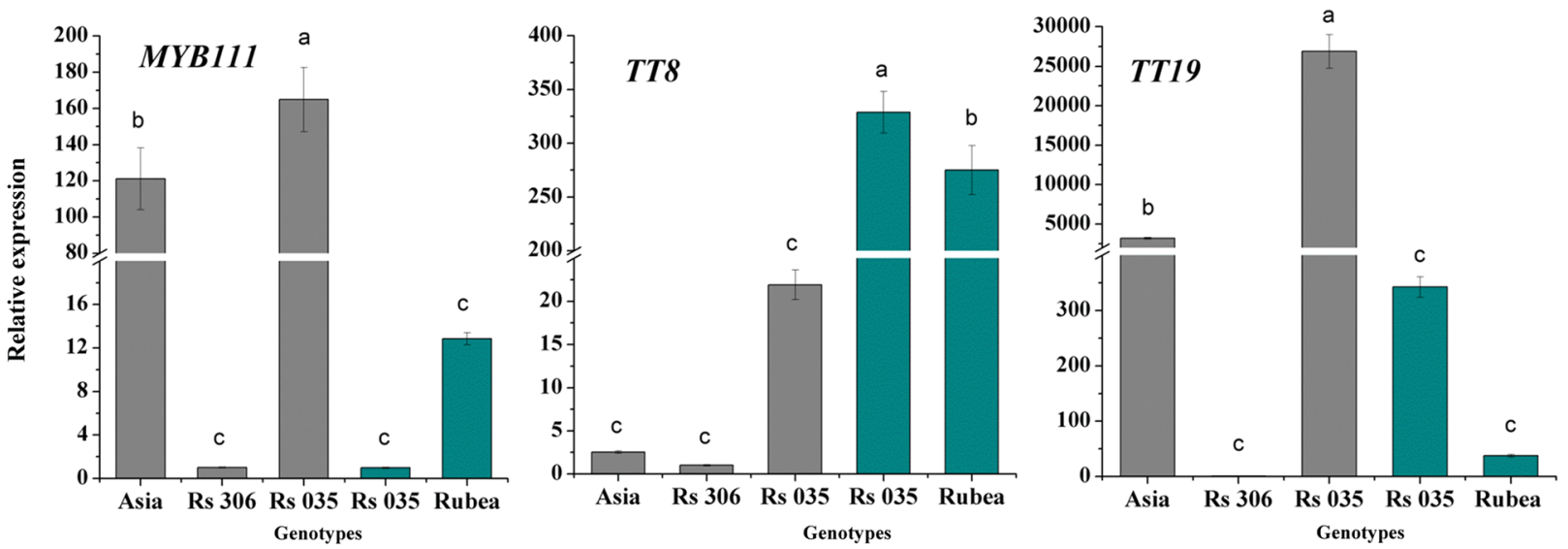

Fig. 4 Expression patterns of the anthocyanin pathway genes. a Upstream structural and biosynthesis genes, $\mathbf{b}$ downstream biosynthesis genes, and $\mathbf{c}$ regulatory and transporter genes of the $A$ and $C$ genomes, determined using qRT-PCR with samples of the 4th leaf of 6-week-old plants of Asia (donor of A genome), Rubea (donor of C genome), and the resynthesized line Rs035 (red phenotype), with Rs306 (green phenotype) as a control. Error bars represent the standard deviation of the means of three independent replicates for each genotype, while different letters indicate significant differences among the genotypes at $p<0.01$, following Tukey's method 


\section{Expression profiling of regulatory and transporter genes}

We next attempted to better understand the molecular mechanisms by which R2R3-MYB, bHLH, and WD40 transcription factors regulate anthocyanin biosynthesis in the line Rs035. To investigate whether any of the regulatory or transporter genes were upregulated to control the expression of the anthocyanin structural genes or affect anthocyanin accumulation, we performed an expression analysis of two independent regulatory R2R3-MYB genes (MYB12 and MYB111), four genes of the MBW complex [the MYBs PAP2 and MYB113, the bHLH TT8, and the WD40 Transparent Testa Glabra 1 (TTG1)], and one transporter gene (TT19) from both the $\mathrm{A}$ and $\mathrm{C}$ genomes. Among these seven genes, MYB12, $P A P 2, M Y B 113$, and TTG1 from the A and $\mathrm{C}$ genome backgrounds of Rs035 did not show any striking difference in expression, although the A genome genes were quantitatively more highly expressed than those of the $\mathrm{C}$ genome, and were even higher than in the A genome donor parental line, Asia (Additional file 5: Figure S3b). In contrast, the regulatory gene $M Y B 111$ and the transporter gene TT19 in A genome background were significantly more highly expressed in Rs035 than MYB111 and TT19 in C genome background (170-fold and 75-fold higher expression, respectively). TT8 of $\mathrm{C}$ genome showed a 15-fold higher expression level than TT8 of A genome in Rs035 (Fig. 4c). In addition, five regulatory and transporter genes, of which four from A genome (MYB12, PAP2, MYB111 and BrTT19) and one from C genome $(T T 8)$ showed transgressive overexpression and two genes (MYB113 and MYB111) of C genome showed transgressively repressed expression in the line Rs035, while one regulatory gene (MYB12) of $\mathrm{C}$ genome showed additive expression in Rs035 (Fig. 4c and Additional file 5: Figure S3b). These results also revealed that the interaction of the $\mathrm{A}$ and $\mathrm{C}$ genomes led to synergistic or additive effects on gene expression, possibly due to the complementary actions of the syntenic homeologous regulatory and transporter genes.

\section{Associations between the constituent anthocyanins and the expression levels of the biosynthetic and regulatory genes}

A principal component analysis of the total and constituent levels of anthocyanins and the expressions of the biosynthesis pathway genes in four contrasting lines (Asia, Rs306, Rs035, and Rubea) led to the identification of five principal components (PCs) with an Eigenvalue greater than unity (data not shown). The first three PCs (PC1, $\mathrm{PC} 2$, and $\mathrm{PC} 3$ ) explained $97 \%$ of the total variance in anthocyanin content $(67.5 \%, 22.5 \%$, and $7 \%$ of the variance was explained by $\mathrm{PC} 1, \mathrm{PC} 2$, and $\mathrm{PC} 3$, respectively; Table 2). The total variation accounted for by PC1 was manifested by the higher positive coefficients of some of the upstream and all of the downstream biosynthesis and regulatory genes, the total anthocyanin content, and the majority of the individual anthocyanin components, versus the higher negative coefficients of two upstream biosynthesis genes and one regulatory gene (Table 2 and Fig. 5). PC1 clearly distinguished the red resynthesized line (Rs035) from the green line (Rs306) and the two parental lines (Asia and Rubea). This distinction corresponded to the highly expressed genes, anthocyanin components, and total anthocyanin contents of the four samples, as evidenced by their mean PC scores and different positions in the PCA biplot (Table 2 and Fig. 5). The highly expressed biosynthetic and regulatory genes were plotted against the total anthocyanin content and most of the anthocyanin components in the high-anthocyanin line, Rs035, as shown in the PCA biplot (Fig. 5).

Meanwhile, PC2 clearly separated the resynthesized line Rs035 from its diploid parents B. rapa (Asia) and B. oleracea (Rubea) in the PCA biplot, along with their respective gene expression patterns, total anthocyanin contents, and anthocyanin compositions (Fig. 5). This was further evidenced by the mean PC scores in the resynthesized lines, Rs035 and Rs306 (+6.45 and -3.31, respectively), and the diploid parental lines, Rubea and Asia ( -3.72 and 0.57 , respectively; Table 2), and by the higher significant positive correlations between total anthocyanin and the gene expression patterns, as observed from the Pearson's correlation analysis (Additional file 3: Table S2).

\section{Discussion}

The anthocyanins are a group of flavonoid secondary metabolites with a wide range of colors, from light yellow to blue or even black. Brassica vegetables have diverse anthocyanin contents; however, very few highanthocyanin cultivars are available for $B$. napus. Previously, only one report has investigated the quantitative trait loci regulating anthocyanin accumulation in this species [14]. Here, we attempted to develop resynthesized $B$. napus lines with enhanced levels of anthocyanin in the leaves by hybridizing high-anthocyanin varieties of B. rapa var. pekinensis (A genome) and B. oleracea var. capitata (C genome) followed by chromosome doubling. Many interspecific hybrids have been developed from various wild and cultivated $\mathrm{A}$ and $\mathrm{C}$ genome plants [40]; however, this is the first report of the hybridization of two anthocyanin-enriched diploid Brassica species. A total of 39 tetraploid hybrids with highly variable total anthocyanin contents were generated (Fig. 2), suggesting recombination between homoeologous chromosomes, and/or altered DNA methylation status of the genes, which could lead to rapid phenotypic changes in 
Table 2 Component loadings and mean principal component (PC) scores, showing the association between the total and constituent anthocyanin contents and the expression levels of the biosynthetic and regulatory genes, as determined using a principal component analysis

\begin{tabular}{|c|c|c|c|}
\hline Variable & PC1 & PC2 & PC3 \\
\hline \multicolumn{4}{|l|}{ Upstream genes } \\
\hline PAL1 & 0.14 & 0.29 & 0.24 \\
\hline PAL2 & -0.06 & 0.36 & 0.26 \\
\hline $\mathrm{C} 4 \mathrm{H}$ & -0.09 & 0.34 & 0.24 \\
\hline $4 C L 1$ & -0.04 & 0.37 & 0.25 \\
\hline $\mathrm{CHS}$ & 0.22 & -0.01 & -0.19 \\
\hline $\mathrm{CHI}$ & 0.23 & 0.01 & -0.09 \\
\hline $\mathrm{F} 3 \mathrm{H}$ & 0.21 & -0.05 & 0.19 \\
\hline$F 3^{\prime} H$ & 0.23 & -0.08 & -0.03 \\
\hline \multicolumn{4}{|l|}{ Downstream genes } \\
\hline$F L S 1$ & 0.23 & 0.02 & 0.11 \\
\hline DFR & 0.23 & -0.03 & 0.15 \\
\hline ANS & 0.23 & -0.08 & 0.12 \\
\hline UGT75C1 & 0.22 & -0.01 & -0.17 \\
\hline UGT78D2 & 0.23 & 0.03 & 0.10 \\
\hline \multicolumn{4}{|l|}{ Regulatory genes } \\
\hline MYB12 & 0.23 & 0.04 & -0.05 \\
\hline MYB111 & 0.22 & -0.03 & -0.22 \\
\hline PAP2 & 0.22 & 0.08 & 0.10 \\
\hline MYB113 & 0.23 & 0.01 & -0.05 \\
\hline BrTT8 & -0.11 & -0.34 & 0.17 \\
\hline TTG1 & 0.20 & 0.11 & -0.15 \\
\hline TT19 & 0.22 & -0.03 & 0.21 \\
\hline \multicolumn{4}{|l|}{ Anthocyanin components } \\
\hline C3-dig5-gl & -0.04 & -0.38 & 0.23 \\
\hline C3-s-dig5-gl & 0.21 & -0.10 & 0.28 \\
\hline C3-sop5-m.gl & 0.23 & -0.01 & -0.07 \\
\hline Mal3-(p-c)gl py de & 0.21 & -0.03 & 0.28 \\
\hline Pe3-(6-ac)-gl & 0.20 & 0.01 & -0.37 \\
\hline C3-(p-c)digl5-gl & -0.12 & -0.33 & 0.15 \\
\hline Total anthocyanin & 0.09 & -0.35 & 0.23 \\
\hline Eigenvalue & 18.22 & 6.07 & 2.10 \\
\hline$\%$ of the variation explained & 67.5 & 22.5 & 7.0 \\
\hline Cumulative $\%$ of the variation explained & 67.5 & 90.0 & 97.0 \\
\hline Genotype & Mean PC score \pm SD & & \\
\hline Asia & $0.57 \pm 0.09 B$ & $0.36 \pm 0.12 B$ & $-2.37 \pm 0.20 C$ \\
\hline Rs306 & $-3.31 \pm 0.02 C$ & $3.28 \pm 0.11 \mathrm{~A}$ & $0.89 \pm 0.09 \mathrm{~A}$ \\
\hline Rs035 & $6.45 \pm 0.04 \mathrm{~A}$ & $-0.29 \pm 0.08 C$ & $0.96 \pm 0.08 \mathrm{~A}$ \\
\hline Rubea & $-3.72 \pm 0.02 \mathrm{D}$ & $-3.35 \pm 0.07 \mathrm{D}$ & $0.52 \pm 0.04 B$ \\
\hline
\end{tabular}

SD standard deviation, C3-dig5-g/ cyanidin 3-diglucoside-5-glucoside, C3-s-dig5-g/ cyanidin 3-(sinapoyl)-diglucoside-5-glucoside, C3-sop5-m.g/ cyanidin 3-sophoroside-5-malonylglucoside, Mal3-(p-c)gl py de malvidin-3-(p-coumaroyl)glucoside pyruvic derivative, Pe3-(6-ac)-gl pelargonidin-3-(6-acetyl)-glucoside, C3-( $p$ - $c)$ dig/5-gl cyanidin 3-(p-coumaroyl) diglucoside-5-glucoside

Letters in mean PC score against the genotypes are mean separation using Tukey test with $p<0.05$

the newly resynthesized allopolyploids [39, 41-47]. Several studies on resynthesized polyploids (including in the genera Arabidopsis, Brassica, Gossypium, Nicotiana, Triticum, and Triticale) and natural polyploid species have demonstrated a variety of genomic changes, including deletions, gene conversions, transposon activations, chromosomal rearrangements, and epigenetic 


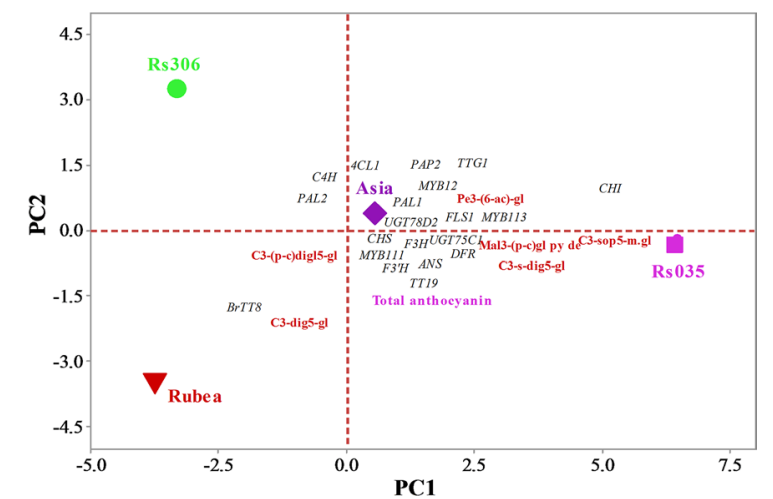

Fig. 5 Biplot of total and constituent anthocyanin contents and the expression of anthocyanin biosynthesis pathway genes in four Brassica lines (Asia, Rubea, Rs035, and Rs306), determined using a principle component analysis (PCA). Genotype text color reflects the visual phenotype of the leaves. Blue and italic texts denote anthocyanin components and gene names, respectively. C3-dig5-gl cyanidin 3-diglucoside-5-glucoside, C3-s-dig5-gl cyanidin 3-(sinapoyl) -diglucoside-5-glucoside, C3-sop5-m.gl cyanidin 3-sophoroside-5-ma lonylglucoside, Mal3-(p-c)gl py de malvidin-3-(p-coumaroyl)glucoside pyruvic derivative, Pe3-(6-ac)-g/ pelargonidin-3-(6-acetyl)-glucoside, C3-(p-c)digl5-gl cyanidin 3-(p-coumaroyl) diglucoside-5-glucoside

modifications, all of which altered the phenotypes of the resulting plants [48-50].

By using HPLC, we identified five anthocyanin compounds in Rs035, whereas only four and three compounds were detected in Asia and Rubea, respectively (Table 1). The additional anthocyanin compound in Rs035 might be the result of recombination and/or a genomic restructuring of the anthocyanin biosynthetic and regulatory genes between its $\mathrm{A}$ and $\mathrm{C}$ genomes. The genomes of allopolyploids can be considerably different from those of the parental genomes [51-53].

We identified 73 and 58 genes involved in the anthocyanin pathway in B. rapa (A genome) and B. oleracea ( $C$ genome), respectively. The A-genome genes were distributed across all 10 A-genome chromosomes (A01$\mathrm{A} 10)$ and the $\mathrm{C}$-genome genes were located on all nine $\mathrm{C}$-genome chromosomes (C01-C09). Some of the genes could not be mapped to chromosomes and were instead mapped to scaffolds; more $\mathrm{C}$-genome than A-genome genes were mapped to these non-contiguous genomic sequences (Additional file 1: Table S1a, Additional file 2: Table S2b and Additional file 5: Figure S2a, b). Similar distributions of anthocyanin genes were previously reported [28], and whole-genome analyses established that the degree of gene density on the chromosomes might be the result of genome triplication events in Brassica $[54,55]$. The genomes of B. rapa and B. oleracea therefore contain more than one copy of most anthocyanin biosynthesis genes. The microsynteny analyses among the anthocyanin biosynthesis and regulatory genes in A. thaliana, B. rapa, and B. oleracea corroborated the close relationships among them, and confirmed their evolution from a common ancestor, consistent with the report on $B r A Q P$ genes by Kayum et al. [56].

Anthocyanins are important secondary metabolites that play a defensive role against a range of abiotic and biotic stresses in plants. Recent studies have also reported multiple benefits of anthocyanin consumption in human health. Despite their importance, the genes involved in anthocyanin biosynthesis and its regulation are poorly understood in B. napus, although they have been well characterized in other Brassicaceae species, including Arabidopsis [57], Chinese cabbage [29], cabbage [11], pak choi (B. rapa ssp. chinensis) [58], kohlrabi [27], radish (Raphanus raphanistrum ssp. sativus) [59], and brown mustard (B. juncea) [60]. Here, we tried to predict the putative candidate gene(s) involved in anthocyanin biosynthesis, using expression profiling to assess the magnitude and nature of their expression patterns. Previous studies reported that MBW transcriptional activation complexes may activate the expression of the anthocyanin biosynthesis genes [23, 25, 61]. Our expression data showed that the biosynthesis genes $P A L, C 4 H, 4 C L, C H S$, $C H I, F 3 H$, and $F 3^{\prime} H$ were differentially expressed among the two parental lines (Asia and Rubea) and the two resynthesized lines (green Rs306 and red Rs035).

Among the upstream genes, A-genome $\mathrm{CHS}$ and $\mathrm{CHI}$ showed 2- and tenfold higher levels of expression, respectively, in Rs035 than the C-genome versions of these genes, while only one C-genome upstream gene, $F 3 H$, showed a 1.5-fold higher expression than the A-genome gene (Fig. 4a). The highly expressed upstream genes seem to be putatively involved in the production of flavonols and other flavonoid compounds in the resynthesized line Rs035. Of the downstream genes, FLS1, DFR, ANS, and UGT75C1 from the A- and C-genomes were differentially expressed in the examined lines; A-genome FLS1, DFR, BrANS, and UGT75C1 had 20-, 60-, 4-, and 55-fold higher expression levels, respectively, in Rs035 than their $\mathrm{C}$-genome counterparts. Highly expressed genes downstream of the anthocyanin biosynthesis pathway may contribute to the accumulation of anthocyanin [62].

Seven upstream (PAL1, PAL2, 4CL1, CHS, CHI, F3H, $F 3^{\prime} H$ ) and four downstream genes (FLS1, DFR, ANS, and UGT75C1) showed transgressive overexpression in Rs035 in comparison with its parents. Most of the upstream and downstream genes of the Rs035 C-genome showed additive expression (Fig. 4a, b and Additional file 5: Figure S3a). The transgressive overexpression of the abovementioned genes might be caused by several mechanisms encountered in the hybrid [63], including the reunion of divergent genomes in the same nucleus, enabling the 
interaction of divergent genes [64] or a dosage effect of the homeologous genes within the same background.

Based on their significantly higher expression levels in one or both genomes of Rs035 in comparison with the parental lines, we predicted that one R2R3-MYB (MYB111) and one bHLH (TT8) of the MBW complex, as well as a transporter gene (TT19), may be responsible for the higher anthocyanin accumulation in Rs035 (Fig. 4c and Additional file 5: Figure S3b). High anthocyanin accumulation in Rs035 might achieved due to combined and complementary effect of those three regulatory and transporter genes by activating the transcription of structural genes, while one regulatory R2R3-MYB (MYB111) as well one transporter gene (TT19) were upregulated in the parent line Asia; one bHLH (TT8) of the MBW complex was upregulated in the parent Rubea. The combined effect of those regulatory and transporter genes might have higher effect on activating the transcription of structural genes in Rs035 than in their parents.

The R2R3-MYB PAP1, MYB113, and MYB114, and the bHLH TT8 are known to be involved in the upregulation of anthocyanin biosynthesis in Arabidopsis leaves [65]. Xie et al. [29] reported that the bHLH-encoding regulatory genes and a large proportion of structural genes are candidates for the accumulation of anthocyanins in $B$. rapa. In $A$. thaliana and a number of other species, MBW complexes were identified as the activators of anthocyanin biosynthesis $[66,67]$. In the resynthesized line Rs035, R2R3-MYB and bHLH transcription factors could bind to specific cis-elements in the promoters of the anthocyanin structural genes; this finding is in agreement with [26]. The transgressive expression of the predicted regulatory and transporter genes in the resynthesized line Rs035 might also be due to interaction of cis- and trans-regulatory factors in the synthetic hybrids as reported by other research efforts [68-70].

Based on our comparative univariate expression data, we identified three early-biosynthesis (CHS, CHI, and $F 3 H$ ), four late-biosynthesis (FLS1, DFR, ANS, and UGT75C1), two transcriptional regulator (MYB111 and TT8), and one transporter (TT19) genes as the putative candidates responsible for the enhanced accumulation of anthocyanins in the resynthesized line Rs035 (Fig. 4a-c and Additional file 5: Figure S3a, b). We deployed a multivariate analytical approach to identify and visualize the overall association of the expression of these genes with the total and constituent anthocyanin contents in the four Brassica lines. The association of the highly expressed genes with the total anthocyanin contents and the anthocyanin components of Rs035 in the PCA biplot indicate that these genes may be responsible for the higher accumulation of anthocyanins in this line. The total anthocyanin contents and the expression of key regulators (MYB111, TT8, and TT19) showed significant positive correlations with the expression levels of the structural genes, which further demonstrated the association between these genes and the total anthocyanin contents. Previous studies indicated that each $B$. rapa and $B$. oleracea chromosome has a homologous counterpart in B. napus [71, 72]; therefore, the predicted A- and $\mathrm{C}$-genome genes are likely confined within the resynthesized $B$. napus. A previous study reported the upregulation of $\mathrm{BrCHS}, \mathrm{BrF} 3 \mathrm{H}, \mathrm{BrANS}, \mathrm{BrDFR}$, and $\mathrm{BrTT}$. in anthocyanin-enriched pak choi [27], while $B o F 3^{\prime} H$, BoMYB2, and BoTT8 were hypothesized to be responsible for anthocyanin accumulation in red cabbage [11]. Our PCA biplot data support the involvement of these genes in the accumulation of high levels of anthocyanin in Rs035 (Fig. 5).

\section{Conclusions}

Anthocyanin-enriched $B$. napus lines were developed by hybridizing anthocyanin-rich varieties of $B$. rapa and B. oleracea. A series of in silico analyses were used to identify and characterize the anthocyanin genes and determine their chromosomal distributions and syntenic relationships. Variable numbers of anthocyanin genes are distributed across all chromosomes of the reconstituted $B$. napus lines, which were inherited from the $\mathrm{A}$ - and $\mathrm{C}$-genome donor parents. Anthocyanin biosynthesis is a complex enzymatic process; therefore, a coordinated analysis of the expression of many anthocyanin biosynthesis-related genes was carried out to elucidate the molecular mechanisms involved, within the limits of the environmental cues that control the pathway and the final accumulation of the end products. An overview of the most highly expressed genes of each of the pathway nodes in our study revealed that the late-biosynthesis genes of the A-genome are comparatively highly expressed in Rs035 line compared with those of the $\mathrm{C}$ genome in the same background, but $\mathrm{A}$ and $\mathrm{C}$ genomes complemented each other. Our expression data also clearly demonstrated that the independent regulator BrMYB111, BolTT8, and the transporter gene $B r T T 19$ are key regulators controlling the production of high levels of anthocyanin in resynthesized $B$. napus, as demonstrated by their transgressive and additive expression patterns. Our study lays the foundation for enabling the development of high-anthocyanin-accumulating cultivars of $B$. napus, with brilliant colors and health-promoting benefits that may be attractive to both growers and consumers. In addition, our resynthesized lines and the differentially expressed genes we have identified could be used to transfer the anthocyanin traits to 
other commercial rapeseed lines using molecular and conventional breeding.

\section{Methods \\ Plant materials and sampling}

Four lines from the genus Brassica were used, including a red-green Chinese cabbage line (B. rapa cv. Asia), a red cabbage line (B. oleracea cv. Rubea), a green resynthesized rapeseed line (allopolyploid B. napus cv. Rs306), and a red resynthesized rapeseed line (allopolyploid B. napus cv. Rs035). The Rs035 line was developed by crossing the inbred Chinese cabbage line $B$. rapa $\mathrm{cv}$. Asia with the cabbage line $B$. oleracea cv. Rubea. The embryos were rescued 18-20 days after crossing and were cultured on Murashige and Skoog (MS) medium. The chromosomes of the developed plants were diploidized using a 4 -hr $0.1 \%$ colchicine root treatment. Successfully crossed hybrid plants were identified using the conserved ortholog set (COS) marker COS1078 [73]. The genotypes of the allopolyploid plants with diploid chromosomes were confirmed using a CyFlow ploidy analyzer and 4',6-diamidino-2-phenylindole (DAPI) solution (Sysmex, Norderstedt, Germany).

\section{RNA extraction and CDNA synthesis}

The fourth leaves of 6-week-old plants were collected and immediately frozen in liquid nitrogen, then stored at $-80^{\circ} \mathrm{C}$. Around $2 \mathrm{~g}$ of each leaf sample was ground into powder for RNA extraction and anthocyanin quantification. Total RNA was isolated from $100 \mathrm{mg}$ of the finely ground powder using a RNeasy Plant Mini Kit (Qiagen, Hilden, Germany), according to the manufacturer's instructions. The RNA was treated with RNase-free DNase I (Qiagen) before cDNA synthesis. The concentration of the extracted RNA was measured using a NanoDrop ND-1000 spectrophotometer (Thermo Fisher Scientific, Waltham, MA, USA). The cDNA was synthesized using a First-Strand cDNA Synthesis kit (Thermo Fisher Scientific) in a $20-\mu \mathrm{l}$ reaction, according to the manufacturer's instructions, then stored at $-20{ }^{\circ} \mathrm{C}$ until required.

\section{Quantification of total anthocyanin content}

The total anthocyanin content was determined following a previously described protocol [74] with some modifications. Three leaves of each genotype were frozen in liquid nitrogen and ground into powder, then $100 \mathrm{mg}$ of each sample was transferred into an Eppendorf tube containing $1 \mathrm{ml}$ acidic methanol $(1 \% \mathrm{HCl}$, w/v). Samples were mixed overnight at room temperature by shaking at $50 \mathrm{rpm}$ in the dark. After that, the mixtures were centrifuged at $12,000 \times g$ for $10 \mathrm{~min}$. The supernatants were collected and the absorbance of each sample was determined at 530 and $657 \mathrm{~nm}$ wavelengths. The total anthocyanin content was computed using the following equation: $\mathrm{Q}_{\text {Anthocyanins }}=\left(\mathrm{A}_{530}-0.25 \times \mathrm{A}_{657}\right) \times \mathrm{FW}^{-1}$, where $\mathrm{Q}_{\text {Anthocyanins }}=$ total anthocyanin content, $\mathrm{A}_{530}=$ absorption at $530 \mathrm{~nm}, \mathrm{~A}_{657}=$ absorption at $657 \mathrm{~nm}$, and FW= fresh weight of leaf samples (g). The total anthocyanins were quantified from five replicates of each biological sample.

\section{Extraction and quantification of anthocyanin compounds using high-performance liquid chromatography}

For the high-performance liquid chromatography (HPLC) analysis, the leaf samples were freeze-dried and ground into a fine powder in liquid nitrogen. A 100-mg sample of each finely powdered leaf was vortexed for $5 \mathrm{~min}$ in $2 \mathrm{ml} \mathrm{5 \%}$ formic acid (v/v) in ultrapure water, after which it was sonicated for $20 \mathrm{~min}$. The samples were centrifuged at 12,000 $\mathrm{rpm}$ for $10 \mathrm{~min}$, then the supernatant was filtered through a $0.45-\mu \mathrm{m}$ PTFE filter (Toyo Roshi Kaisha, Ltd., Tokyo, Japan). The extracted anthocyanins were quantified using an Agilent 1200 series HPLC (Agilent Technologies, Santa Clara, CA, USA), and the chromatographic separation was carried out using reverse phase columns (particle size $4 \mu \mathrm{m}$; C18 $80 \mathrm{~A}, 250 \times 4.60 \mathrm{~mm}$; Phenomenex, Torrence, CA, USA) at a temperature of $40{ }^{\circ} \mathrm{C}$. The injection volume was $10 \mu \mathrm{l}$ with a flow rate of $1 \mathrm{ml} \mathrm{min}{ }^{-1}$. Elution was performed using mobile phase A ( $5 \%$ formic acid) and B (acetonitrile), with the following gradient program: 0-8 min using $5-10 \% \mathrm{~B} ; 8-15 \mathrm{~min}$ at $13 \% \mathrm{~B} ; 15-18 \mathrm{~min}$ using $15 \% \mathrm{~B}$ and kept constant until $25 \mathrm{~min}$. After $25 \mathrm{~min}$, the concentration of B was quickly decreased to $5 \%$ and kept constant until $35 \mathrm{~min}$. Detection was carried out at a wavelength of $520 \mathrm{~nm}$. Individual anthocyanin components were quantified by comparing the area under their HPLC peak with that of a known standard (cyanidin-3-0-glucoside), and expressed as $\mathrm{mg} \mathrm{g}^{-1}$ dry weight.

\section{Selection and in silico analysis of anthocyanin biosynthesis genes}

The anthocyanin pathway genes of the $\mathrm{A}$ and $\mathrm{C}$ genomes were collected from the BRAD database (http://brass icadb.org/brad/) using a syntenic gene search of the anthocyanin pathway genes in A. thaliana retrieved from TAIR (https://www.arabidopsis.org/). These genes were also checked against the Bolbase (http://www.ocrigenomics.org/bolbase/genes.htm) and EnsemblPlants (http://plants.ensembl.org/) databases. Finally, a complementary method, Hidden Markov Models profiling, was performed to increase the accuracy of the gene identification in the B. rapa and B. oleracea genomes. In addition, 
the primary gene features (gene structure, distribution on chromosomes, gene position, strand, protein length, molecular weight, and iso-electric point) were analyzed using ExPasy (http://au.expasy.org/tools/pi_tool.html). The physical positions of the genes were drafted onto the A- and C-genome chromosomes from $B$. rapa and B. oleracea, respectively, using Map Chart v.2.2 (http:// www.wageningenur.nl/en/show/Mapchart.htm). The microsyntenic relationships of the anthocyanin structural, regulatory, and transporter genes in A. thaliana, $B$. rapa, and $B$. oleracea were plotted using circos software (http://circos.ca/) [75].

\section{Expression analysis using quantitative real-time (qRT) PCR} Gene expression levels were analyzed using qRT-PCR performed on a Roche LightCycler ${ }^{\circledR} 96$ System (Roche Applied Science, Penzberg, Germany). Gene-specific primers (Additional file 4: Table S3a, b) were designed using an online tool, Primer3Plus [76], and were targeted to the gene sequences collected from BRAD [77] and Bolbase [55]. The 10- $\mu$ l reaction mixture contained $5 \mu \mathrm{l}$ of $2 \times$ qPCRBIO SyGreen Mix Lo-ROX (PCR Biosystems, London, UK), $1 \mu \mathrm{l}$ of each gene-specific forward (F) and reverse (Rv) primer (10 pmol), $2 \mu \mathrm{l}$ distilled deionized water $\left(\mathrm{ddH}_{2} \mathrm{O}\right)$, and $1 \mu \mathrm{l}$ of $80 \mathrm{ng} \mathrm{ll}^{-1}$ cDNA as a template. The qRT-PCR was performed under the following conditions: initial denaturation at $95{ }^{\circ} \mathrm{C}$ for $5 \mathrm{~min}$; followed by 50 cycles of $95^{\circ} \mathrm{C}$ for $10 \mathrm{~s}$, $58^{\circ} \mathrm{C}$ for $10 \mathrm{~s}$, and $72{ }^{\circ} \mathrm{C}$ for $15 \mathrm{~s}$. Three replicates of each sample were analyzed. The data were processed using the LightCycler 96 SW 1.1 software and quantified using the Cq value of each qRT-PCR amplicon, following the $2^{-\Delta \Delta \mathrm{Ct}}$ method [78]. The data were normalized against the mean amplicon value of the three $A C T I N$ genes (as internal controls) expressed by $B$. rapa and B. oleracea.

\section{Statistical analysis}

The total and constituent anthocyanin contents and the gene expression levels were analyzed using one-way analysis of variance (ANOVA) and Tukey's pair-wise comparisons in Minitab v.17 (Minitab Inc., State College, PA, USA). The data were presented as the mean of three replicates \pm standard deviation $( \pm S D)$. Data used for principal component analyses (PCAs) were standardized by subtracting the mean and dividing the result by the standard deviation. The constituent anthocyanins determined using HPLC and the relative gene expression levels calculated from the qRT-PCR were set as variables for the PCA using Minitab v.17 (Minitab Inc.).

\section{Additional files}

Additional file 1: Table S1a. In silico analysis of Anthocyanin genes identified in B. rapa with their Arabidopsis orthologs and biological activity.

Additional file 2: Table S1 b. In silico analysis of anthocyanin genes identified in B. oleracea with their Arabidopsis orthologs and biological activity.

Additional file 3: Table S2. Pearson's correlation analysis among the expression of the anthocyanin biosynthesis pathway genes of A- and C-genome along with anthocyanin components in the four contrasting Brassica lines.

Additional file 4: Table S3a. Oligonucleotide primers used for qRT-PCR analysis of B. rapa. Table S3b. Oligonucleotide primers used for qRT-PCR analysis of B. oleracea.

Additional file 5: Figure S1. Morphological distinctiveness and anthocyanin contents of four Brassica lines. The visual phenotype (a) and 4th-leaf total anthocyanin contents of 6-week-old plants (b) of the parental lines Asia (B. rapa) and Rubea (B. oleracea), the red allopolyploid resynthesized $B$. napus (Rs035), and the control green allopolyploid resynthesized $B$. napus (Rs306) are shown. Figure S2. Chromosomal distributions of the putative anthocyanin biosynthesis genes in (a) Brassica rapa and (b) Brassica oleracea. The chromosome number is indicated at the top of each chromosome. The scale (left) is in megabases (Mb). The colors represent the putative structural genes of the phenylpropanoid pathway (black), early-biosynthesis (red), late-biosynthesis (green), positive-regulatory (blue), and negative-regulatory (brown) genes. Genes assigned to scaffold sequences are not shown on the physical maps. Figure S3. Relative gene expression of anthocyanin pathway genes which did not show striking expression changed compared among the studied four lines, (a) structural and (b) regulatory and transporter genes.

\section{Abbreviations}

4CL: 4-coumaratel-coA ligase; ANOVA: analysis of variance; ANS: anthocyanidin synthase; bHLH: basic helix loop helix; Bolbase: Brassica oleracea Genomics Database; BRAD: Brassica Database; C4H: cinnamate-4-hydroxylase; cDNA: complementary DNA; CHI: chalcone isomerase; CHS: chalcone synthase; COS: conserved ortholog set; DFR: dihydroflavanol reductase; F3H: flavanone-3-hydroxylase; FLS: flavonol synthase; FW: fresh weight; HPLC: high performance liquid chromatography; MS: Murashige and Skoog; MYB: myeloblastosis family of transcription factors; PAL: phenylalanine ammonia lyase; PCA: principal component analysis; Pi: isoelectric point; qPCR: quantitative polymerase chain reaction; Rs: resynthesised; RT-PCR: reverse transcription polymerase chain reaction; SD: standard deviation; TAIR: the arabidopsis information resource; TT: transparent testa; TTG1: transparent testa glabra 1; UGT: uradin glycosyltransferase.

\section{Authors' contributions}

I-SN, J-IP, and UKN formulated the concept and designed the research. GG performed the research, analyzed the real-time expression data, and prepared the tables and figures. UKN conducted the statistical analysis and wrote the manuscript. MRH conducted the PCA and polished the manuscript. MKB and $\mathrm{H}$-TK performed the in silico analysis. HRK conducted the HPLC analysis. All authors read and approved the final manuscript.

\section{Author details}

${ }^{1}$ Department of Horticulture, Sunchon National University, 255 Jungang-ro, Suncheon, Jeonnam 57922, South Korea. ${ }^{2}$ Department of Genetics and Plant Breeding, Bangladesh Agricultural University, Mymensingh, Bangladesh.

${ }^{3}$ University-Industry Cooperation Foundation, Sunchon National University, 255 Jungang-ro, Suncheon, Jeonnam 57922, South Korea. ${ }^{4}$ Plant Systems Engineering Research Center, Korea Research Institute of Bioscience and Biotechnology, Daejeon, South Korea.

\section{Acknowledgements}

Special thanks to Dr. Christian Möllers, Department of Crop Science, GeorgAugust University, Göttingen, Germany, for providing the Rs306 seed. 


\section{Competing interests}

The authors declare that they have no competing interests.

\section{Availability of data and materials}

We declare that the dataset(s) supporting the conclusions of this article are included within the article and its Additional files 1,2,3,4,5.

\section{Consent for publication}

Not applicable.

\section{Ethics approval and consent to participate} Not applicable.

\section{Funding}

This research was supported by the Golden Seed Project (Center for Horticultural Seed Development, No. 213007-05-3-CG100); the Ministry of Agriculture, Food and Rural Affairs (MAFRA); the Ministry of Oceans and Fisheries (MOF); the Rural Development Administration (RDA); and the Korea Forest Service (KFS), South Korea.

\section{Publisher's Note}

Springer Nature remains neutral with regard to jurisdictional claims in published maps and institutional affiliations.

Received: 18 July 2018 Accepted: 9 November 2018 Published online: 26 November 2018

\section{References}

1. Steyn W, Wand S, Holcroft D, Jacobs G. Anthocyanins in vegetative tissues: a proposed unified function in photoprotection. New Phytol. 2002;155:349-61.

2. Lin-Wang K, Bolitho K, Grafton K, Kortstee A, Karunairetnam S, McGhie TK, et al. An R2R3 MYB transcription factor associated with regulation of the anthocyanin biosynthetic pathway in Rosaceae. BMC Plant Biol. 2010;10:50.

3. Buer CS, Imin N, Djordjevic MA. Flavonoids: new roles for old molecules. J Integr Plant Biol. 2010;52:98-111.

4. Wang H, Fan W, Li H, Yang J, Huang J, Zhang P. Functional characterization of dihydroflavonol-4-reductase in anthocyanin biosynthesis of purple sweet potato underlies the direct evidence of anthocyanins function against abiotic stresses. PLoS ONE. 2013;8:e78484.

5. Pourcel L, Routaboul J-M, Cheynier V, Lepiniec L, Debeaujon I. Flavonoid oxidation in plants: from biochemical properties to physiological functions. Trends Plant Sci. 2007:12:29-36.

6. Nakabayashi R, Yonekura-Sakakibara K, Urano K, Suzuki M, Yamada Y, Nishizawa T, Matsuda F, Kojima M, Sakakibara H, Shinozaki K. Enhancement of oxidative and drought tolerance in Arabidopsis by overaccumulation of antioxidant flavonoids. Plant J. 2014;77:367-79.

7. Lev-Yadun S, Gould KS. Role of anthocyanins in plant defence. Anthocyanins. New York: Springer; 2008. p. 22-8

8. Mazza G, Kay CD, Cottrell T, Holub BJ. Absorption of anthocyanins from blueberries and serum antioxidant status in human subjects. J Agric Food Chem. 2002;50:7731-7.

9. He Q, Zhang Z, Zhang L. Anthocyanin accumulation, antioxidant ability and stability, and a transcriptional analysis of anthocyanin biosynthesis in purple heading Chinese cabbage (Brassica rapa L. ssp. pekinensis). J Agric Food Chem. 2015;64:132-45.

10. Thomasset S, Teller N, Cai H, Marko D, Berry DP, Steward WP, Gescher AJ. Do anthocyanins and anthocyanidins, cancer chemopreventive pigments in the diet, merit development as potential drugs? Cancer Chemother Pharmacol. 2009;64:201-11.

11. Yuan Y, Chiu L-W, Li L. Transcriptional regulation of anthocyanin biosynthesis in red cabbage. Planta. 2009;230:1141.

12. Tedesco I, Russo GL, Nazzaro F, Russo M, Palumbo R. Antioxidant effect of red wine anthocyanins in normal and catalase-inactive human erythrocytes. J Nutr Biochem. 2001;12:505-11.
13. Fujii K, Ohmido N. Stable progeny production of the amphidiploid resynthesized Brassica napus cv. Hanakkori, a newly bred vegetable. Theor Appl Genet. 2011;123:1433-43.

14. Li H, Zhu L, Yuan G, Heng S, Yi B, Ma C, Shen J, Tu J, Fu T, Wen J. Fine mapping and candidate gene analysis of an anthocyanin-rich gene, BnaA. PL1, conferring purple leaves in Brassica napus L. Mol Genet Genomics. 2016:291:1523-34.

15. Petrussa E, Braidot E, Zancani M, Peresson C, Bertolini A, Patui S, Vianello A. Plant flavonoids-biosynthesis, transport and involvement in stress responses. Int J Mol Sci. 2013;14:14950-73.

16. Dixon DP, Skipsey M, Edwards R. Roles for glutathione transferases in plant secondary metabolism. Phytochemistry. 2010;71:338-50.

17. Mueller LA, Goodman CD, Silady RA, Walbot V. AN9, a petunia glutathione $S$-transferase required for anthocyanin sequestration, is a flavonoidbinding protein. Plant Physiol. 2000;123:1561-70.

18. Holton TA, Cornish EC. Genetics and biochemistry of anthocyanin biosynthesis. Plant Cell. 1995;7:1071.

19. Winkel-Shirley B. Flavonoid biosynthesis. A colorful model for genetics, biochemistry, cell biology, and biotechnology. Plant Physiol. 2001:126:485-93.

20. Tanaka Y, Sasaki N, Ohmiya A. Biosynthesis of plant pigments: anthocyanins, betalains and carotenoids. Plant J. 2008;54:733-49.

21. Petroni K, Tonelli C. Recent advances on the regulation of anthocyanin synthesis in reproductive organs. Plant Sci. 2011;181:219-29.

22. Hichri I, Barrieu F, Bogs J, Kappel C, Delrot S, Lauvergeat V. Recent advances in the transcriptional regulation of the flavonoid biosynthetic pathway. J Exp Bot. 2011;62:2465-83.

23. Broun P. Transcriptional control of flavonoid biosynthesis: a complex network of conserved regulators involved in multiple aspects of differentiation in Arabidopsis. Curr Opin Plant Biol. 2005;8:272-9.

24. Lepiniec L, Debeaujon I, Routaboul J-M, Baudry A, Pourcel L, Nesi N, Caboche M. Genetics and biochemistry of seed flavonoids. Annu Rev Plant Biol. 2006:57:405-30.

25. Koes R, Verweij W, Quattrocchio F. Flavonoids: a colorful model for the regulation and evolution of biochemical pathways. Trends Plant Sci. 2005; 10:236-42.

26. Lang D, Weiche B, Timmerhaus G, Richardt S, Riaño-Pachón DM, Corrêa LG, Reski R, Mueller-Roeber B, Rensing SA. Genome-wide phylogenetic comparative analysis of plant transcriptional regulation: a timeline of loss, gain, expansion, and correlation with complexity. Genome Biol Evol. 2010;2:488-503.

27. Zhang Y, Hu Z, Zhu M, Zhu Z, Wang Z, Tian S, Chen G. Anthocyanin accumulation and molecular analysis of correlated genes in purple Kohlrabi (Brassica oleracea var. gongylodes L.). J Agric Food Chem. 2015;63:4160-9.

28. Guo N, Cheng F, Wu J, Liu B, Zheng S, Liang J, Wang X. Anthocyanin biosynthetic genes in Brassica rapa. BMC Genomics. 2014;15:426.

29. Xie L, Li F, Zhang S, Zhang H, Qian W, Li P, Zhang S, Sun R. Mining for candidate genes in an introgression line by using RNA sequencing: the anthocyanin overaccumulation phenotype in Brassica. Front Plant Sci. 2016;7:1245.

30. Jackson S, Chen ZJ. Genomic and expression plasticity of polyploidy. Curr Opin Plant Biol. 2010;13:153-9.

31. Zhang J, Zhang J, Liu W, Han H, Lu Y, Yang X, Li X, Li L. Introgression of Agropyron cristatum 6P chromosome segment into common wheat for enhanced thousand-grain weight and spike length. Theor Appl Genet. 2015;128:1827-37.

32. Wang Y, Zhang Z, Jia L, Li Z, Li J, Lou Q, Chen J. Molecular and cytogenetic analyses provide evidence of the introgression of chromosomal segments from the wild Cucumis hystrix into the cultivated cucumber through the bridge of a synthetic allotetraploid. Mol Breed. 2017;37:89.

33. Nagahara U. Genomic analysis of Brassica with special reference to the experimental formation of B. napus and peculiar mode of fertilization. Jpn J Bot. 1935;7:389-452

34. Rana D, Boogaart T, O'neill CM, Hynes L, Bent E, Macpherson L, Park JY, Lim YP, Bancroft I. Conservation of the microstructure of genome segments in Brassica napus and its diploid relatives. Plant J. 2004:40:725-33.

35. Cheung F, Trick M, Drou N, Lim YP, Park J-Y, Kwon S-J, Kim J-A, Scott R, Pires JC, Paterson AH. Comparative analysis between homoeologous genome segments of Brassica napus and its progenitor species reveals extensive sequence-level divergence. Plant Cell. 2009;21:1912-28. 
36. Xiong Z, Pires JC. Karyotype and identification of all homoeologous chromosomes of allopolyploid Brassica napus and its diploid progenitors. Genetics. 2011;187:37-49.

37. Song K, Lu P, Tang K, Osborn TC. Rapid genome change in synthetic polyploids of Brassica and its implications for polyploid evolution. Proc Natl Acad Sci USA. 1995;92:7719-23.

38. Gaeta RT, Chris Pires J. Homoeologous recombination in allopolyploids: the polyploid ratchet. New Phytol. 2010;186:18-28.

39. Szadkowski E, Eber F, Huteau V, Lode M, Huneau C, Belcram H, Coriton O, Manzanares-Dauleux M, Delourme R, King GJ. The first meiosis of resynthesized Brassica napus, a genome blender. New Phytol. 2010;186:102-12.

40. Song K, Osborn TC, Williams PH. Brassica taxonomy based on nuclear restriction fragment length polymorphisms (RFLPS). Theor Appl Genet. 1990;79:497-506

41. Gaeta RT, Pires JC, Iniguez-Luy F, Leon E, Osborn TC. Genomic changes in resynthesized Brassica napus and their effect on gene expression and phenotype. Plant Cell. 2007;19:3403-17.

42. Lukens LN, Pires JC, Leon E, Vogelzang R, Oslach L, Osborn T. Patterns of sequence loss and cytosine methylation within a population of newly resynthesized Brassica napus allopolyploids. Plant Physiol. 2006;140:336-48.

43. Szadkowski E, Eber F, Huteau V, Lodé M, Coriton O, Jenczewski E, Chevre A. Polyploid formation pathways have an impact on genetic rearrangements in resynthesized Brassica napus. New Phytol. 2011;191:884-94.

44. Xu Y, Zhong L, Wu X, Fang X, Wang J. Rapid alterations of gene expression and cytosine methylation in newly synthesized Brassica napus allopolyploids. Planta. 2009;229:471-83.

45. Wang H, Jiang J, Chen S, Qi X, Fang W, Guan Z, Teng N, Liao Y, Chen F. Rapid genetic and epigenetic alterations under intergeneric genomic shock in newly synthesized Chrysanthemum morifolium $\times$ Leucanthemum paludosum hybrids (Asteraceae). Genome Biol Evol. 2014;6:247-59.

46. Li X, Guo W, Wang B, Li X, Chen H, Wei L, Wang Y, Wu J, Long H. Instability of chromosome number and DNA methylation variation induced by hybridization and amphidiploid formation between Raphanus sativus $\mathrm{L}$. and Brassica alboglabra Bailey. BMC Plant Biol. 2010;10:207.

47. Zhang X, Liu T, Li X, Duan M, Wang J, Qiu Y, Wang H, Song J, Shen D. Interspecific hybridization, polyploidization, and backcross of Brassica oleracea var. alboglabra with B. rapa var. purpurea morphologically recapitulate the evolution of Brassica vegetables. Sci Rep. 2016;6:18618.

48. Comai L. The advantages and disadvantages of being polyploid. Nat Rev Genet. 2005;6:836.

49. Chen ZJ. Genetic and epigenetic mechanisms for gene expression and phenotypic variation in plant polyploids. Annu Rev Plant Biol. 2007;58:377-406.

50. Doyle JJ, Flagel LE, Paterson AH, Rapp RA, Soltis DE, Soltis PS, Wendel JF. Evolutionary genetics of genome merger and doubling in plants. Annu Rev Genet. 2008;42:443-61.

51. Comai L. Genetic and epigenetic interactions in allopolyploid plants. Plant Mol Biol. 2000;43:387-99.

52. Zhao X, Si Y, Hanson RE, Crane CF, Price HJ, Stelly DM, Wendel JF, Paterson $\mathrm{AH}$. Dispersed repetitive DNA has spread to new genomes since polyploid formation in cotton. Genome Res. 1998;8:479-92.

53. Volkov RA, Borisjuk NV, Panchuk II, Schweizer D, Hemleben V. Elimination and rearrangement of parental rDNA in the allotetraploid Nicotiana tabacum. Mol Biol Evol. 1999;16:311-20.

54. Wang X, Wang H, Wang J, Sun R, Wu J, Liu S, Bai Y, Mun J-H, Bancroft I, Cheng F. The genome of the mesopolyploid crop species Brassica rapa. Nat Genet. 2011:43:1035.

55. Yu J, Zhao M, Wang X, Tong C, Huang S, Tehrim S, Liu Y, Hua W, Liu S. Bolbase: a comprehensive genomics database for Brassica oleracea. BMC Genomics. 2013;14:664.

56. Kayum MA, Park J-I, Nath UK, Biswas MK, Kim H-T, Nou I-S. Genome-wide expression profiling of aquaporin genes confer responses to abiotic and biotic stresses in Brassica rapa. BMC Plant Biol. 2017;17:23.
57. Shi M-Z, Xie D-Y. Biosynthesis and metabolic engineering of anthocyanins in Arabidopsis thaliana. Recent Pat Biotechnol. 2014;8:47-60.

58. Zhang L, Xu B, Wu T, Yang Y, Fan L, Wen M, Sui J. Transcriptomic profiling of two Pak Choi varieties with contrasting anthocyanin contents provides an insight into structural and regulatory genes in anthocyanin biosynthetic pathway. BMC Genomics. 2017;18:288.

59. Lim S-H, Song J-H, Kim D-H, Kim JK, Lee J-Y, Kim Y-M, Ha S-H. Activation of anthocyanin biosynthesis by expression of the radish R2R3-MYB transcription factor gene RsMYB1. Plant Cell Rep. 2016;35:641-53.

60. Xie Q, Hu Z, Zhang Y, Tian S, Wang Z, Zhao Z, Yang Y, Chen G. Accumulation and molecular regulation of anthocyanin in purple tumorous stem mustard (Brassica juncea var. tumida Tsen et Lee). J Agric Food Chem. 2014;62:7813-21.

61. Dixon RA, Xie DY, Sharma SB. Proanthocyanidins-a final frontier in flavonoid research? New Phytol. 2005;165:9-28.

62. Owens DK, Alerding AB, Crosby KC, Bandara AB, Westwood JH, Winkel BS. Functional analysis of a predicted flavonol synthase gene family in Arabidopsis. Plant Physiol. 2008;147:1046-61.

63. Hegarty MJ, Barker GL, Wilson ID, Abbott RJ, Edwards KJ, Hiscock SJ. Transcriptome shock after interspecific hybridization in Senecio is ameliorated by genome duplication. Curr Biol. 2006;16:1652-9.

64. Riddle NC, Birchler JA. Effects of reunited diverged regulatory hierarchies in allopolyploids and species hybrids. Trends Genet. 2003;19:597-600.

65. Gonzalez A, Zhao M, Leavitt JM, Lloyd AM. Regulation of the anthocyanin biosynthetic pathway by the TTG1/bHLH/Myb transcriptional complex in Arabidopsis seedlings. Plant J. 2008;53:814-27.

66. Zhang F, Gonzalez A, Zhao M, Payne CT, Lloyd A. A network of redundant bHLH proteins functions in all TTG1-dependent pathways of Arabidopsis. Development. 2003:130:4859-69.

67. Feller A, Machemer K, Braun EL, Grotewold E. Evolutionary and comparative analysis of MYB and bHLH plant transcription factors. Plant J. 2011;66:94-116.

68. Zhuang $Y$, Adams KL. Extensive allelic variation in gene expression in Populus F1 hybrids. Genetics. 2007;177:1987-96.

69. Chaudhary B, Flagel L, Stupar RM, Udall JA, Verma N, Springer NM, Wende JF. Reciprocal silencing, transcriptional bias and functional divergence of homeologs in polyploid cotton (Gossypium). Genetics. 2009;182:503-17.

70. Tirosh I, Reikhav S, Levy AA, Barkai N. A yeast hybrid provides insight into the evolution of gene expression regulation. Science. 2009;324:659-62.

71. Jiang C, Ramchiary N, Ma Y, Jin M, Feng J, Li R, Wang H, Long Y, Choi SR, Zhang C. Structural and functional comparative mapping between the Brassica A genomes in allotetraploid Brassica napus and diploid Brassica rapa. Theor Appl Genet. 2011;123:927.

72. Chalhoub B, Denoeud F, Liu S, Parkin IA, Tang H, Wang X, Chiquet J, Belcram H, Tong C, Samans B. Early allopolyploid evolution in the postneolithic Brassica napus oilseed genome. Science. 2014;345:950-3.

73. Jeong Y-M, Chung W-H, Chung H, Kim N, Park B-S, Lim K-B, Yu H-J, Mun $\mathrm{J}-\mathrm{H}$. Comparative analysis of the radish genome based on a conserved ortholog set (COS) of Brassica. Theor Appl Genet. 2014;127:1975-89.

74. Mehrtens F, Kranz H, Bednarek P, Weisshaar B. The Arabidopsis transcription factor MYB12 is a flavonol-specific regulator of phenylpropanoid biosynthesis. Plant Physiol. 2005;138:1083-96.

75. Krzywinski M, Schein J, Birol I, Connors J, Gascoyne R, Horsman D, Jones SJ, Marra MA. Circos: an information aesthetic for comparative genomics. Genome Res. 2009;19:1639-45.

76. Untergasser A, Nijveen H, Rao X, Bisseling T, Geurts R, Leunissen JA. Primer3Plus, an enhanced web interface to Primer3. Nucleic Acids Res. 2007;35:W71-4.

77. Cheng F, Liu S, Wu J, Fang L, Sun S, Liu B, Li P, Hua W, Wang X. BRAD, the genetics and genomics database for Brassica plants. BMC Plant Biol. 2011;11:136.

78. Livak KJ, Schmittgen TD. Analysis of relative gene expression data using real-time quantitative PCR and the $2^{-\triangle \Delta C T}$ method. Methods. 2001;25:402-8 